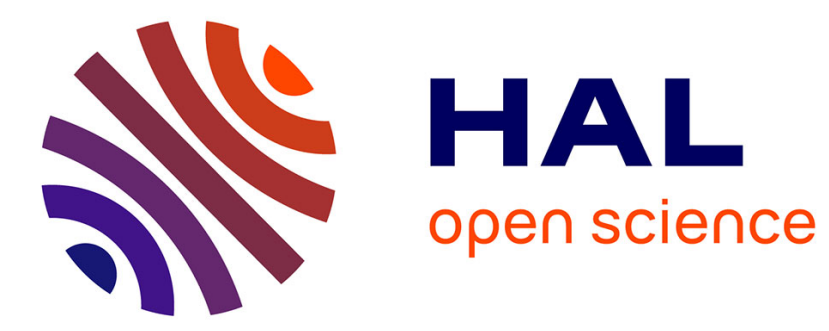

\title{
Kinetic Accessibility of Porous Material Adsorption Sites Studied through the Lattice Boltzmann Method
}

Jean-Mathieu Vanson, François-Xavier Coudert, Michaela Klotz, Anne Boutin

\section{To cite this version:}

Jean-Mathieu Vanson, François-Xavier Coudert, Michaela Klotz, Anne Boutin. Kinetic Accessibility of Porous Material Adsorption Sites Studied through the Lattice Boltzmann Method. Langmuir, 2017, 33 (6), pp.1405 - 1411. 10.1021/acs.langmuir.6b04472 . hal-01685631

\section{HAL Id: hal-01685631 \\ https://hal.science/hal-01685631}

Submitted on 24 Apr 2019

HAL is a multi-disciplinary open access archive for the deposit and dissemination of scientific research documents, whether they are published or not. The documents may come from teaching and research institutions in France or abroad, or from public or private research centers.
L'archive ouverte pluridisciplinaire HAL, est destinée au dépôt et à la diffusion de documents scientifiques de niveau recherche, publiés ou non, émanant des établissements d'enseignement et de recherche français ou étrangers, des laboratoires publics ou privés. 


\title{
Kinetic accessibility of porous materials adsorption sites studied through Lattice Boltzmann method
}

\author{
Jean-Mathieu Vanson, ${ }^{\dagger}$ François-Xavier Coudert, ${ }^{*} \uparrow$ Michaela Klotz, ${ }^{\ddagger}$ and Anne Boutin ${ }^{*, \dagger}$ \\ $\dagger$ École Normale Supérieure, PSL Research University, Département de Chimie, Sorbonne Universités - \\ UPMC Univ Paris 06, CNRS UMR 8640 PASTEUR, 24 rue Lhomond, 75005 Paris, France \\ $\ddagger$ Laboratoire de Synthèse et Fonctionnalisation des Céramiques, UMR 3080 Saint-Gobain CREE/CNRS, \\ 550 Avenue Alphonse Jauffret, 84306 Cavaillon, France
}

ฯPSL Research University, Chimie ParisTech, CNRS, Institut de Recherche de Chimie Paris, 75005 Paris, France

E-mail: fx.coudert@chimie-paristech.fr anne.boutin@ens.fr

\begin{abstract}
We present here a computational model based on the Lattice Boltzmann scheme to investigate the accessibility of active adsorption sites in hierarchical porous materials to adsorbates in a flowing liquid. By studying the transport and adsorption of tracers after they enter in the pore space of the virtual sample, we characterize their kinetics as they pass through the pore space and adsorb on the solid-liquid interface. The model is validated on simple geometries with known analytical solution. We then use it to investigate the influence of regular grooves or disordered roughness on the walls of a slit pore geometry, looking at the impact on adsorption and transport. In particular, we highlight the importance of adsorption site accessibility, which depends on shape and connectivity of the pore space as well as the fluid flow profile and velocity.
\end{abstract}

\section{Introduction}

Materials with hierarchical porosity, ranging from nanometers to micrometers, are of interest for a wide range of industrial applications, because they can potentially combine high specific surface area and high permeability, i.e. low resistance to fluid flow. Such applications typically rely on the host-guest surface interactions, for example in phase separation, gas mixture separation, or ions exchange and capture. In the liquid phase, practical applications at large scale include, for example, water decontamination and removal of pollutants such as heavy metals or radioactive ions.

Understanding the physical and chemical phenomena involved in fluid transport and adsorption in hierarchical porous materials is crucial for the optimization of existing materials and the design of new materials. In particular, the optimization of the pore space (pore dimensions and pore geometry) for a chosen application is a great challenge. The study of transport and adsorption in hierarchical porous materials raises several open fundamental questions. One of them deals with the kinetics of exchange between interconnected pores of different sizes - between macropores and mesopores, or between mesopores and micropores. The influence of the macroporosity on the accessibility of species to the mesoporosity is both a very fundamental question and yet one directly relevant to applications. It depends in nontrivial ways on the geometry of the macropores and mesopores, as well as the topology and interconnectivity of the two pore networks, each of these factors affecting the overall performance of the material.

Despite its fundamental importance and impact, the pub- lished literature is relatively scarce on investigations of kinetic accessibility of adsorption sites in porous materials with hierarchical pore networks. The problem of fluid flow in complex geometries and chromatographic systems has been widely addressed by computational studies, including many based on the Lattice Boltzmann method.1 3 . However, the question of adsorption/desorption and kinetics of tracers has been less studied. One possible approach to this problem has recently been proposed by Boţan et. al., who developed a lattice-based bottom-up model of adsorption and transport in multiscale porous media ${ }^{4}$ by relying on a local thermodynamic description.

We describe here another approach, building upon a Lattice Boltzmann model recently extended to incorporate an accurate description of adsorption of solutes at the fluidsolid interface. ${ }^{\sqrt[56]{6}}$ However, this model cannot in itself answer the question of the accessibility, as it describes the steady state of fluid flow and solute dynamics. In this work, we present an extension of the Lattice Boltzmann model to evaluate the accessibility of active sites by simulating a system out of the steady state. We illustrate it on the kinetics of adsorption on a pore model of a crenelated slit pore, i.e. a larger main pore interconnected with smaller side pockets.

\section{Methods}

The method used in this work is based on a well-known numerical simulation scheme for fluid dynamics, namely the Lattice Boltzmann (LB) model. ${ }^{7}$ The Lattice Boltzmann model finds its roots in the 1980s and has several advantages compared to other computational fluid dynamic methods, making it suitable to address systems at scales intermediate between atomistic or coarse grained simulations (at smaller scale) and finite element-based computational fluid dynamics (at larger scale), as shown on Figure 1

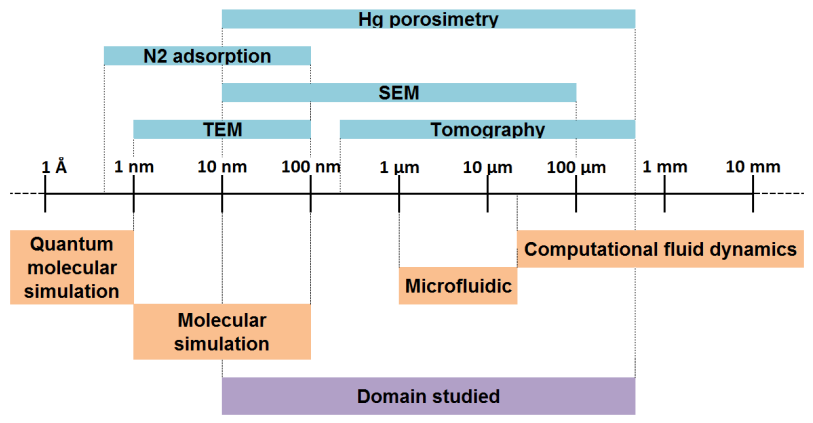

Figure 1. Experimental and modelisation techniques employed for porous materials characterization. 
In particular, the relative simplicity of the computational method make it easy to implement and to parallelize. The LB method works through the propagation of the oneparticle velocity distribution function $f(\boldsymbol{r}, \boldsymbol{c}, t)$ equivalent to the probability of a particle to be at position $r$ with velocity $c$ at time $t$. For numerical integration, time, space and velocities are all discretized. The distribution function is propagated in time through the following equation:

$$
\begin{aligned}
& f_{i}\left(\boldsymbol{r}+\boldsymbol{c}_{\boldsymbol{i}} \Delta t, t+\Delta t\right) \\
& =f_{i}(\boldsymbol{r}, t)+\frac{\left(f_{i}^{e}(\boldsymbol{r}, t)-f_{i}(\boldsymbol{r}, t)\right)}{\tau}+F_{i}^{\text {ext }}
\end{aligned}
$$

where $f_{i}$ is the component of $f$ on velocity vector $i$, i.e. $f_{i}(\boldsymbol{r}, t)=f\left(\boldsymbol{r}, \boldsymbol{c}_{i}, t\right) . f_{i}^{e}$ corresponds to the local MaxwellBoltzmann equilibrium distribution, $\tau$ is the relaxation time, and $F_{i}^{\text {ext }}$ accounts for external forces acting on the liquid and responsible for the fluid flow. This equation is implemented following the method of Ladd and Verberg, ${ }^{8}$ relevant for simulations of fluid dynamics in porous materials. To simulate the dynamical properties of solute dispersed in the fluid we use the moment propagation method proposed by Lowe and Frenkel ${ }^{910}$ and further validated by Merks et al. ${ }^{[1]} \mathrm{A}$ quantity $\boldsymbol{P}(\boldsymbol{r}, t)$ is defined on the lattice and propagated following:

$$
\begin{aligned}
\boldsymbol{P}(\boldsymbol{r}, t+\Delta t)= & \sum_{i} \boldsymbol{P}\left(\boldsymbol{r}-\boldsymbol{c}_{\boldsymbol{i}} \Delta t, t\right) p_{i}\left(\boldsymbol{r}-\boldsymbol{c}_{\boldsymbol{i}} \Delta t, t\right) \\
& +\boldsymbol{P}(\boldsymbol{r}, t)\left(1-\sum_{i} p_{i}(\boldsymbol{r}, t)\right)
\end{aligned}
$$

where $p_{i}(\boldsymbol{r}, t)$ corresponds to the probability of leaving node $\boldsymbol{r}$ with velocity $\boldsymbol{c}_{i}$ :

$$
p_{i}(\boldsymbol{r}, t)=\frac{f_{i}(\boldsymbol{r}, t)}{\rho(\boldsymbol{r}, t)}-w_{i}+w_{i} \lambda \quad \text { with } \quad \lambda=\frac{2 D_{b}}{v_{T}^{2} \Delta t}
$$

Here $\rho$ is the fluid density, $w_{i}$ are constant weights of the velocity model (we chose in this work the D3Q19 model for discretization of velocities), $D_{b}$ is the diffusion coefficient of the tracers in the fluid in bulk phase, and $v_{T}$ is the fluid's speed of sound $\left(v_{T}^{2}=\frac{1}{3} \Delta x^{2} / \Delta t^{2}\right.$, with $\Delta x$ the lattice spacing).

In the published literature, there are relatively few computational lattice-based fluid dynamics models that explicitly take into account the dispersion of solutes and their adsorption at fluid-solid interfaces. $\frac{12}{23}$ In this work, we account for adsorption at the solid-liquid interface by following the technique developped by Levesque et $a l^{[5}$ and refined in later work from our group,,$[24$ — accounting for adsorption and desorption in a generic lattice Boltzmann scheme, by modelling these processes through kinetic rates of adsorption and desorption taking place at interfacial lattice nodes. The adsorption of tracers in the fluid occurs on fluid nodes having at least one solid node as a neighbour. It is described using the adsorbed density of tracers $D_{\text {ads }}$, which is defined only on adsorption sites, and the free density of tracers $D_{\text {free }}$, defined everywhere in the fluid. The adsorption kinetic is described by three physical parameters: the adsorption coefficient $K_{a}$, the desorption coefficient $K_{d}$, and the saturation coefficient $D_{\max }$ (or maximal uptake of a fluid node). The balance between adsorbed and free density is integrated numerically in

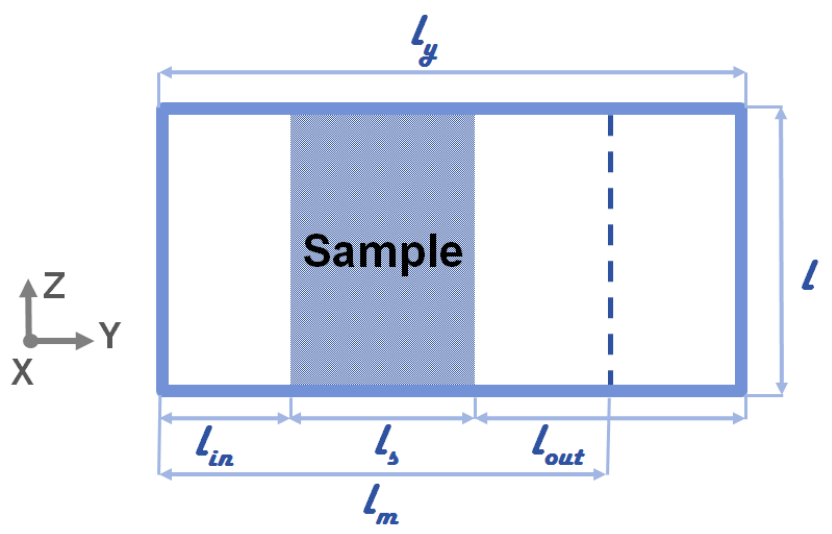

Figure 2. Schematic representation of the simulation box used in this work.

time following first-order kinetics:

$$
\begin{aligned}
D_{\text {ads }}(\boldsymbol{r}, t+\Delta t)= & {\left[1-\frac{D_{\mathrm{ads}}(\boldsymbol{r}, t)}{D_{\max }}\right] D_{\text {free }}(\boldsymbol{r}, t) p_{a} } \\
& +D_{\text {ads }}(\boldsymbol{r}, t)\left(1-p_{d}\right) \\
D_{\text {free }}(\boldsymbol{r}, t+\Delta t)= & D_{\text {free }}(\boldsymbol{r}, t)\left[1-p_{a}+p_{a} \frac{D_{\text {ads }}(\boldsymbol{r}, t)}{D_{\max }}\right] \\
& +D_{\text {ads }}(\boldsymbol{r}, t) p_{d}
\end{aligned}
$$

where $p_{a}=K_{a} \Delta t / \Delta x$ and $p_{d}=K_{d} \Delta t$.

In the simulations reported in this work, a slab of porous material (our "sample") is placed within a bigger simulation box, whose dimension is larger along the direction of fluid flow (see Figure 2). This leads to the presence of two fluid reservoirs, one upstream from the porous matrix, and the other one downstream. We consider a no slip boundary condition at the fluid/solid interface, and periodic boundary conditions at four faces of the simulation box (in the $x y$ and $y z$ planes). However, the periodic boundary conditions are removed in the moment propagation on the two faces perpendicular to the flow (in the $x z$ plane). This general approach is relatively simple to implement, and has been used and validated once in earlier literature for lattice-based computational fluid dynamics of flow, dispersion and catalysis in a packed bed of catalytic spherical particles. 25

In order to compute the transient kinetics of the tracers in the fluid we proceeded to modify the initialization of the free density at time $t=0$. The free density is homogeneously initialized on the upstream face of the simulation box perpendicular to the flow, i.e. the free density is initialized to a constant value on all fluid nodes in the plane $y=0$ when the flux is applied along the $y$ direction, while the initial free density on all other fluid nodes is set to zero.

\section{Practical details}

The data shown here are obtained in a laminar flow regime. We use periodic boundary conditions on two axes ( $x$ and $z$ ), and no slip boundary conditions at the liquid/solid interface for the Lattice Boltzmann scheme. We used a convergence criterion of $10^{-14}$ (in relative step-to-step variation) for the average velocity of the fluid along the three directions of space. The Lattice Boltzmann scheme employed here works in reduced units $(\Delta x, \Delta t, \Delta p)$. The method to switch between reduced units and SI units is available in 


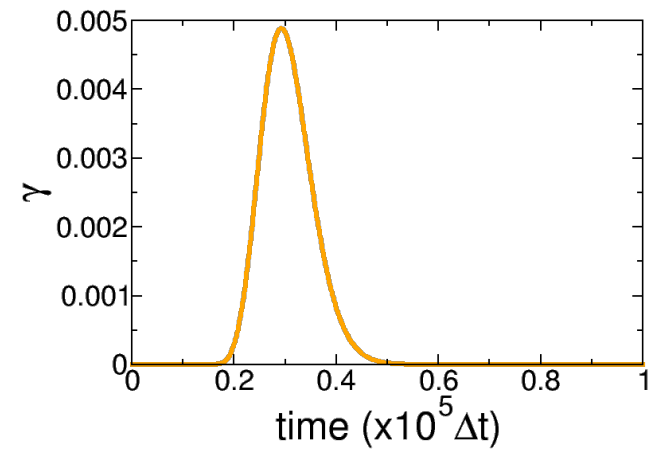

Figure 3. Example of an elution curve $\gamma(t)$ for a pore with slit geometry, with $l=50 \Delta x, l_{m}=800 \delta x, K_{a}=0$ and $F_{\text {ext }}=$ $2 E-6 \Delta p / \Delta x$.

supplementary information. Throughout the simulations we fixed the bulk diffusion coefficient $\left(D_{b}=6.0410^{-8} \mathrm{~m}^{2} \cdot \mathrm{s}^{-1}\right)$, the kinematic viscosity $\left(\nu=10^{-6} \mathrm{~m}^{2} \cdot \mathrm{s}^{-1}\right)$, the density of the fluid $\left(\rho=1000 \mathrm{~kg} \cdot \mathrm{m}^{-3}\right)$, and the density of the solid $\left(\rho_{s}=4970 \mathrm{~kg} \cdot \mathrm{m}^{-3}\right)$.

We used the open-source Lattice Boltzmann code laboetie, 5 available online at https://github.com/ maxlevesque/laboetie. Simulations reported in this work were performed with a modified version of the code, available at https://github.com/maxlevesque/laboetie/ tree/jmvanson (commit 809d0b7). Input files and additional information can be found at our group's data repository, at https://github.com/fxcoudert/citable-data

\section{Model validation}

This section is devoted to the validation of the modified moment propagation scheme, without periodic boundary condition in the flow direction. The key quantity for this is the dispersion coefficient $K$, which represents the spreading of the species inside the material, accounting for both advection and diffusion. It characterizes the influence of the material on the motion of species. In a periodic system it can be computed from the velocity auto-correlation function $Z$ as:

$$
K=\int_{0}^{\infty}[Z(t)-Z(\infty)] \mathrm{d} t
$$

However, in the specific case of our modified technique with no periodic boundary conditions in one direction, we validate the model by computing the dispersion coefficient using a macroscopic procedure common in the field of chromatography. ${ }^{26]}$ At a given position $y=l_{m}$ in the column (see Fig. 2], we measure the flux of tracers passing through the section as a function of time:

$$
\gamma(t)=\sum_{i=1}^{l_{x}} \sum_{k=1}^{l_{z}} D_{\text {free }}\left(i, l_{m}, k, t\right)
$$

The evolution of $\gamma$ with time is the familiar elution curve, an example of which is represented on Figure 3 for a pore with slit geometry, a height of $l=50 \Delta x$, where $l_{m}$ has been set to $800 \delta x$, with no adsorption $\left(K_{a}=0\right)$ and $F_{\text {ext }}=2 E-$ $6 \Delta p / \Delta x$.

Once the simulation has finished and the elution curve is obtained, we fit it to a mathematical model. An impressive number of functions have been proposed in the literature to fit elution curves, and a review on the topic counts up to

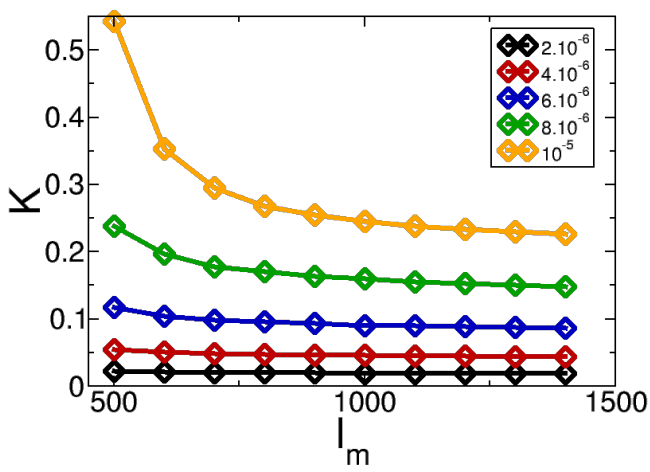

Figure 4. Evolution of the dispersion coefficient $K$ as a function of $l_{m}$ for different values of $F_{\text {ext }}\left(210^{-6}, 410^{-6}, 610^{-6}, 810^{-6}\right.$ $\left.1010^{-6} \Delta p / \Delta x\right)$ in a slit pore geometry, with $l=50 \Delta x, K_{a}=0$.'

90 different mathematical functions used for this purpose. 27 Here, we have chosen simplicity and fit elution curves with a Gaussian function:

$$
f(t)=A e^{\frac{-\left(t-t_{R}\right)^{2}}{2 \sigma_{t}^{2}}}
$$

The Gaussian is the most widely used model in the literature, and our goal here is not to test in detail the influence of one model or another, but to validate our moment propagation scheme. The results obtained do not depend much on the shape of the fitting function, as we only use the peak position and half-maximum width. From the Gaussian fit, we then calculate the Height Equivalent to a Theoretical Plate (HETP), $H$, with the following equation: 28

$$
H=\frac{l_{m} \sigma_{t}^{2}}{t_{R}^{2}}
$$

where $\sigma_{t}$ is the width of the Gaussian fit and $t_{R}$ is the elution time (time $t$ at which $\gamma(t)$ is maximal). Finally, the dispersion coefficient $K$ is computed using the HETP as: $26 \mid 29$

$$
K=\frac{\left\langle v_{y}\right\rangle H}{2}=\frac{\left\langle v_{y}\right\rangle l_{m} \sigma_{t}^{2}}{2 t_{R}^{2}}
$$

where $\left\langle v_{y}\right\rangle$ corresponds to the average velocity of the tracers in the fluid.

We can see in the simplest test case, that of a slit pore with no adsorption $\left(K_{a}=0\right)$ (Fig. 4), that the value of the dispersion coefficient measured through the above procedure is clearly dependent on the position of the measurement (the value of $l_{m}$ ) and converges at high $l_{m}$. This converged value, obtained far from the sample, increases with external force $F_{\text {ext }}$ as is expected. We then compare the values of the dispersion coefficients thus obtained with the analytical solution, which is known for a pore with slit geometry (of width $\left.l_{h}\right): \frac{516}{5}$

$$
\frac{K}{D_{b}}=1-F_{a}+\operatorname{Pe}^{2}\left(\frac{102 \alpha^{2}+18 \alpha+1}{210(1+2 \alpha)^{3}}+\frac{D_{b}}{l_{h}^{2} K_{d}} \frac{2 \alpha}{(1+2 \alpha)^{3}}\right)
$$

where $\mathrm{Pe}=l_{h}\langle v\rangle / D_{b}$ is the Péclet number and $\alpha=$ $K_{a} / K_{d} h$.

Figure 5 compares the values obtained from our model with the analytical solution, for cases without adsorption and in presence of adsorption (panels a and b respectively). We can see that the results agree reasonably well with analytical values, especially considering the numerous assumptions made in the indirect "macroscopic" determination (in particular the Gaussian fit). The difference between indirect 

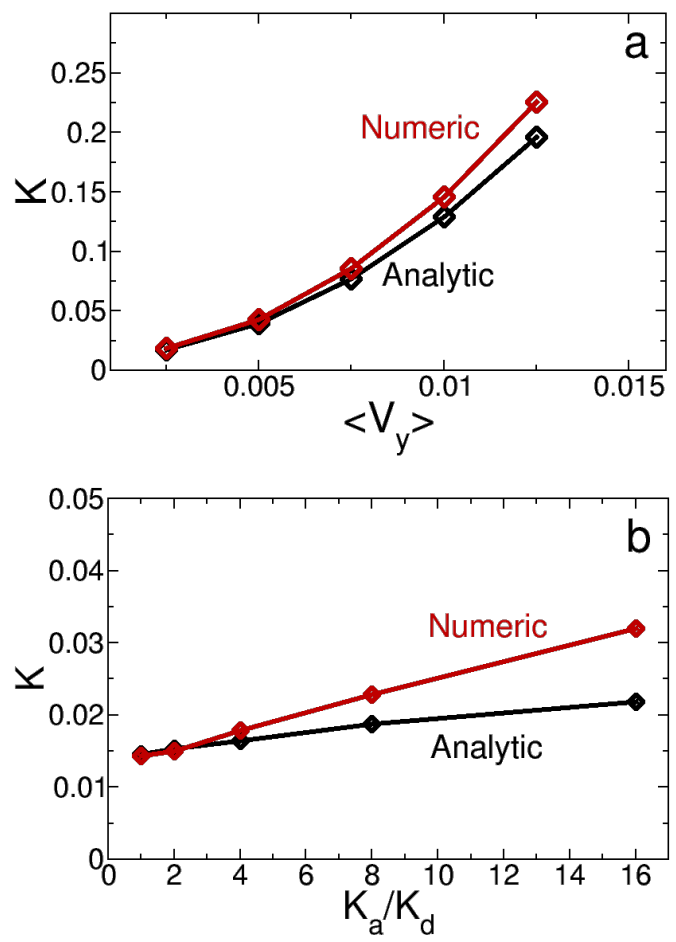

Figure 5. Comparaison of the dispersion coefficient computed numerically from our model and the known analytical solution. (a) As a function of the average velocity of the fluid on $y$ direction, in the absence of adsorption $\left(K_{a}=0\right) . l_{x}=1 \Delta x, l_{y}=1450 \Delta x$, $l_{z}=52 \Delta x, l_{m}=1400 \Delta x$. (b) In presence of adsorption, as a function of the ratio between the adsorption coefficient $K_{a}$ and the desorption coefficient $K_{d}$, with $F_{\text {ext }}=510^{7} \mathrm{~Pa} \cdot \mathrm{m}^{-1}$. Other parameters are: $l_{x}=1 \Delta x, l_{y}=1450 \Delta x, l_{z}=52 \Delta x, l_{m}=1400 \Delta x$

and analytical results increases with the velocity of the fluid due to the fact that the convergence of $K$ with $l_{m}$ is slower at high value of the fluid velocity. This difference also increases with the ratio $K_{a} / K_{d}$ because we employed a simplified Van Deemter equation (Eq. 9 .

\section{Results and discussion}

In this section, we use the extended Lattice Boltzmann model to characterize the evolution with time of the tracers' density inside the fluid and adsorbed at the fluid-solid interface. This yields useful information about the tracer kinetic and the accessibility of the active adsorption sites in two different geometries.

\subsection{Crenelated pore}

We first study the kinetics of solute transport and adsorption in a model geometry of a slit pore with crenels. The geometry is characterized by the aspect ratio of the crenels (or grooves), i.e. the ratio $r$ between the crenel depth and crenel width. Figure 6 shows the time evolution of the density of tracers in the fluid in a slit pore with grooves having an aspect ratio of $r=1$ (whose depth and width are equal). Three snapshots of the density are taken at three moments along the simulation, as the "pulse" of tracers, starting from the left side of the picture, moves towards the right side following the fluid flow. We can see that in this case, the tracers' density follows the profile of the fluid flow, with faster flow

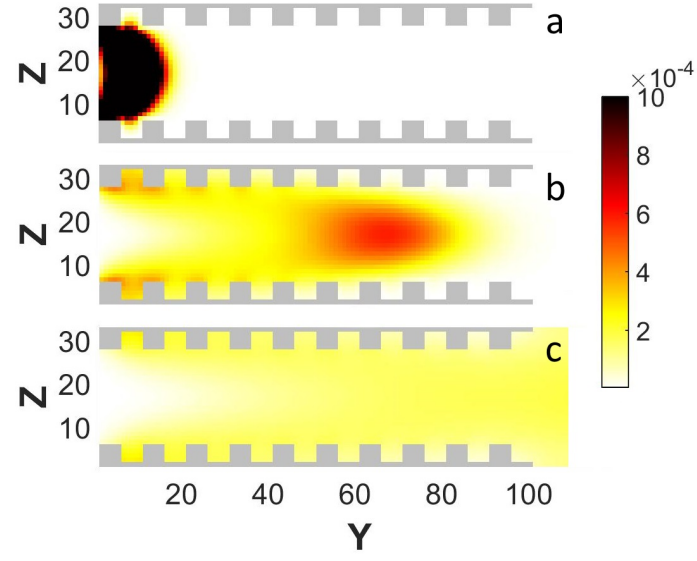

Figure 6. Time evolution of the density of a pulse of tracers in a crenelated pore with liquid flow (flow from left to right). Aspect ratio of the crenels $r=1$, with $h=5, w=5$, and $l=20$. a: $t=1000 \Delta t$; b: $t=10000 \Delta t$; c: $t=20000 \Delta t$. Other parameters of the simulation are: $l_{x}=1 \Delta x, l_{y}=120 \Delta x, l_{z}=32 \Delta x, F_{\text {ext }}=10^{9}$ Pa.m ${ }^{-1}, K_{a}=6.04 \mathrm{~m} . \mathrm{s}^{-1}, K_{d}=6.0410^{6} \mathrm{~s}^{-1}, \Delta x=100 \mathrm{~nm}$.

in the center of the pore. We can also observe the effect of adsorption at the solid-liquid interface on Fig. 6p, where it seems clear that the solute has access to the whole internal area of the pores (i.e., that all adsorption sites are active within the timeframe of the simulation).

We depict the time evolution of tracers in a slit pore with deeper crenels ( $r=4$, all other parameters being equal) on Figure 7. In contrast with the previous case (aspect ratio $r=1$ ), it is clear that for the deeper crenels the tracers do not have access to the entirety of the pores' internal surface. With the chosen flow, it is clear that advection along the $y$ axis is too fast compared to the diffusion of the tracers along the perpendicular $x$ and $z$ axes, which would give them access to the bottom of the grooves. Thus, a dead volume exits in the material, and not all adsorption sites are active in these given conditions. This result parallels what we have seen in earlier work, ${ }^{6]}$ in which we studied the velocity profile for fluid flow in crenelated pore systems and showed the existence of "dead volume", i.e. a non negligible part of the crenel where the velocity of the fluid is close to zero. We observe that this dead volume, which can depend on the velocity of the fluid, becomes significant for $r>1$, when the grooves' depth is larger than their width, as the fluid flow cannot enter the channel.

To quantify this effect we have plotted in Figure 8 the evolution of the adsorbed fraction as a function of simulation time, for various values of crenels' aspect ratio $r$. In all cases, we see that the adsorbed fraction (i.e., the adsorbate uptake) goes through a maximum as a function of time, then decays at long times. The maximum corresponds to the moment when the "cloud" of tracers goes through the sample. For $r<1$ the maximum of the adsorbed fraction increase clearly with $r$ : there, all the adsorption sites are accessible to the adsorbate, and the adsorbed fraction is proportional to the specific surface area, which itself grows as a function of $r$ (since we keep the distance between the top of the crennels constant). For $r>1$, on the other hand, the adsorbed fraction is lower than for $r<1$. Even though, the total specific surface area increases, the adsorbed fraction decreases because not all the sites are accessible for a given fluid velocity. This highlights the fact that, for an adsorbent material to perform best under fluid flow, it is not merely 

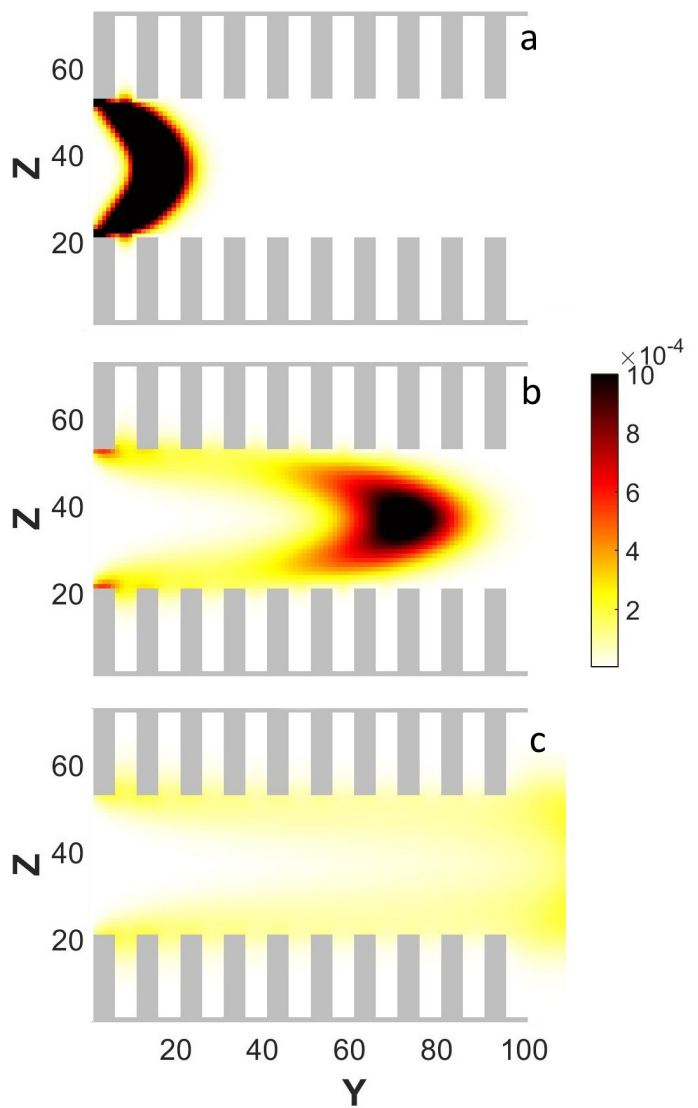

Figure 7. Time evolution of the density of a pulse of tracers in a crenelated pore with liquid flow (flow from left to right). Aspect ratio of the crenels $r=4$, with $h=20, w=5$, and $l=20$ a: $t=1000 \Delta t$, b: $t=5000 \Delta t, \mathrm{c}: t=10000 \Delta t$. Other parameters of the simulation are the same as in Fig. 6 .

dictated by the absolute specific surface area, but also by its accessibility in specific conditions, as the system is not at thermodynamic equilibrium (as is often considered the case for gas adsorption) or in a steady state.

\subsection{Random roughness}

The crenels studied in the previous section can be considered as an ordered "roughness" or small-scale disorder on the surface of the walls of a slit pore. Here, we extend this study by looking at the impact of a disordered (or "random") roughness of the adsorbent walls on the kinetics of tracers in the fluid. The process by which our rough slit pore models were generated - which relies on aggregation of smaller particles onto pre-existing walls - is described in Supporting Information. The roughness is controlled by the parameter controlling the aggregation, $A_{c}$, so that decreasing values of $A_{c}$ lead to increase in the roughness of the walls.

Figure 9 presents a timelapse of 3D views of the advection and adsorption of a pulse of tracers in a randomly aggregated slit pore. The tracers start at $t=0$ on the left side of the sample (upstream), and the fluid flow goes from left to right. As in the simpler geometry, the tracers follows the fluid flow profile, with higher density at the center of the pore, and lower close to the solid-liquid interface. At long times (see $t=9000$ on Fig. 90 we observe a direct effect of adsorption on the distribution of tracers, as the tracer density is lower downstream than upstream - in spite of the fact that the

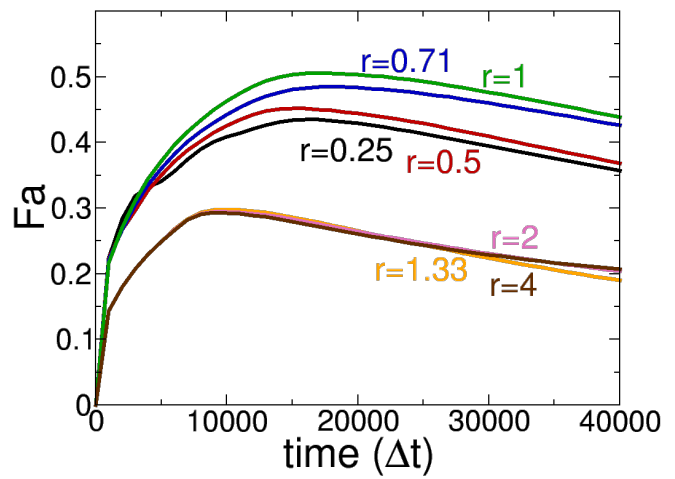

Figure 8. Effect of the ratio $r$ between the height and the width of the crenels on fraction adsorbed. The simulation parameters are the same as in Fig. 7

adsorption and desorption coefficients are identical for all adsorption sites. A large amount of solute is adsorbed upstream at the solid-liquid interface, thus the density of free tracers decreases close to the interface. There is therefore a lower concentration of available tracers on the adsorption sites located downstream, the adsorbed density is thus lower.

Quantitatively, we show on Figure 10 the maximum value of the adsorbed fraction over time (the peak value of the elution curve, $\max \gamma(t))$ as a function of the external force $F_{\text {ext }}$. The different values of aggregation criterion $A_{c}$ represent three different values of the roughness, where $A_{c}=1 / 8$ corresponds to the lowest roughness coefficient and $A_{c}=1 / 32$ to the highest roughness coefficient. In all cases, we observe that $F_{a}$ decreases when $F_{\text {ext }}$ increases i.e. the fraction adsorbed is lower for high velocities of the fluid. The tracers close to the interface are adsorbed at the entrance of the material leading to a lower density close to the adsorption sites. Then, the tracers do not have time to diffuse perpendicularly to the flow to reach the active sites. This effect increase with fluid velocity at constant bulk diffusion coefficient and is, in a broader fashion, dependent on the Péclet number of the fluid flow.

We can see that, in the case of random heterogeneous roughness, we do not observe the effect seen in the ordered crenels: as roughness increases, most adsorption sites remain active and thus higher roughness coefficient does not yield to a lower fraction adsorbed (as was the case for crenels). That is because the "side pockets" of the slit pore created by our aggregation procedure are well-connected to the central area of the slit pore, and have an effective aspect ratio (width over depth) that corresponds to the $r<1$ case in the crenelated slit pore. We thus see that having disordered multiscale porosity can in this case be more efficient, in terms of fluid transport and solute adsorption, than a well-ordered porous space of similar dimensions.

\section{Conclusion}

We have here extended and applied a Lattice Boltzmann scheme for fluid transport in nanoporous materials, taking into account adsorption of solutes on the solid-liquid interface. We use it to study the kinetics of tracer adsorption and the accessibility of adsorption sites in simple models of hierarchical (or multi-scale) porosity. We validated this model on a simple slit pore geometry, then used it to study a slit pore with grooves (or side pockets) of various aspect 

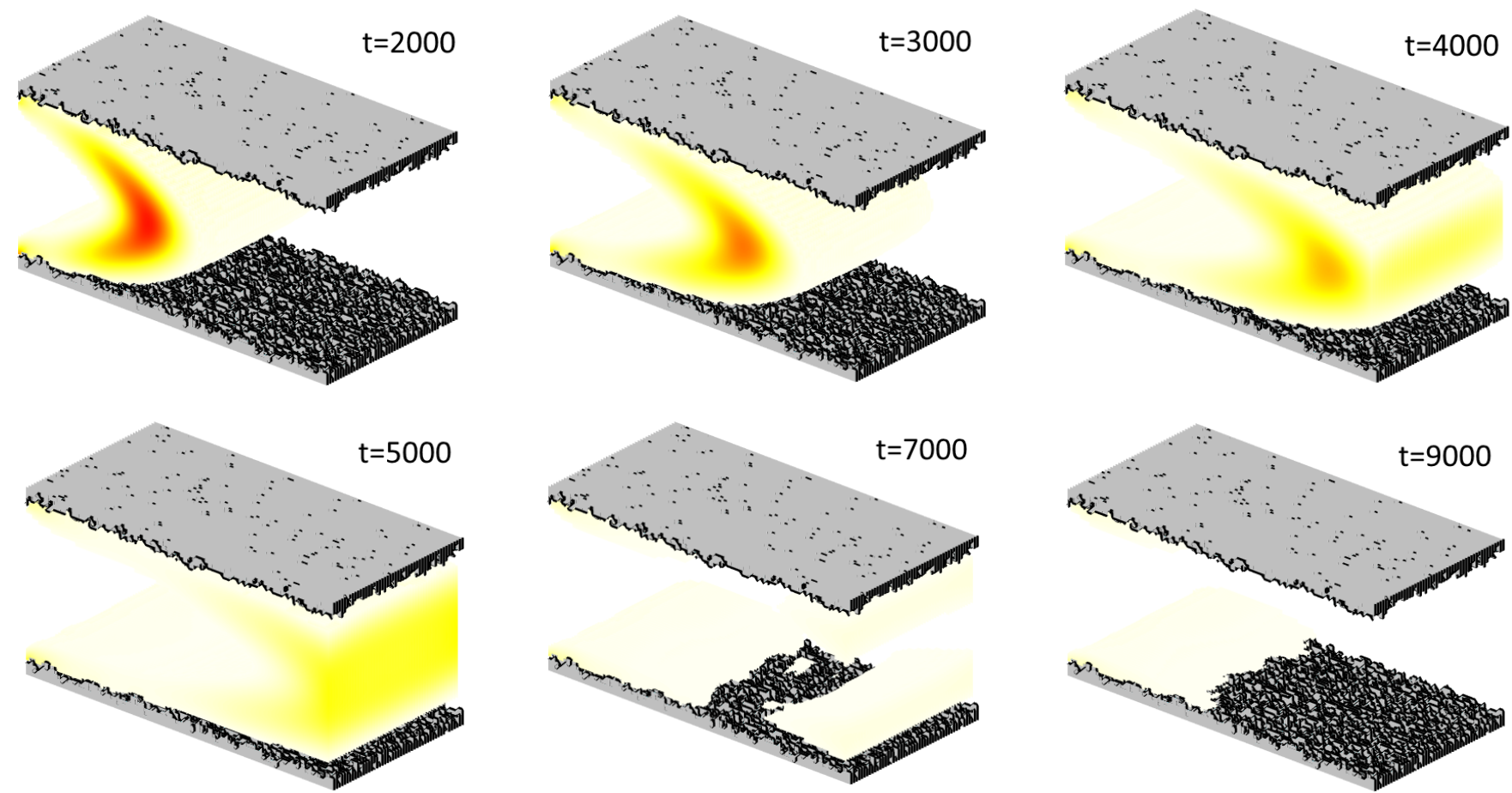

Figure 9. 3D view of spatial repartition of Free density in slit pore geometry with random roughness on the walls. The units of time is the reduced ones $(\Delta t)$. Input parameters for the simulations: $l_{x}=50 \Delta x, l_{y}=150 \Delta x, l_{z}=52 \Delta x, F_{\text {ext }}=10^{9} \mathrm{~Pa} \cdot \mathrm{m}^{-1}, K_{a}=6.04 \mathrm{~m} . \mathrm{s}^{-1}$, $K_{d}=6.0410^{6} \mathrm{~s}^{-1}, \Delta x=100 \mathrm{~nm}$.

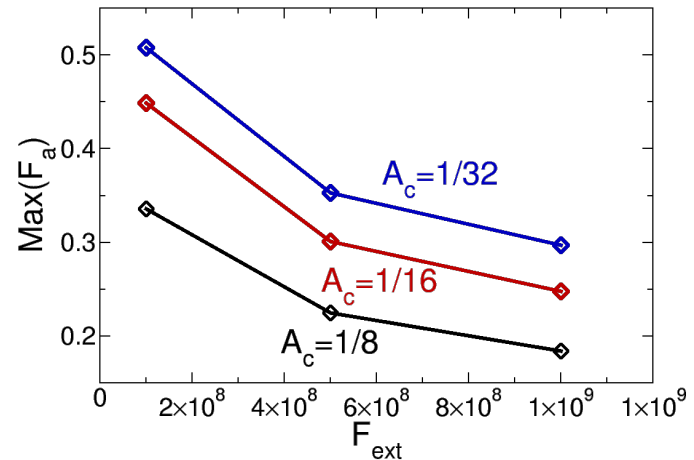

Figure 10. Maximum values of the fraction adsorbed as a function of $F_{\text {ext }}$ for different roughness. The simulation parameters are the same as in Fig. 9

ratio. We demonstrate the potential limitations due to inacessibility of some adsorption sites in the presence of deep grooves, highlighting the problem of access of the solute to the mesoporosity (here crenels) in the walls of the material in presence of flow. We then studied the effect of a more disordered type of porosity, through computer-generated models of rough slit pores with controlled rugosity. This opens the way to future work on complex, realistic models of hierarchical porous materials, e.g. coming from reconstructed 3D images obtained by X-ray tomography.

\section{Supporting Information}

The Supporting Information is available free of charge on the ACS Publications website. Description of the random roughness aggregation model. Relations between reduced units and SI units.

\section{Acknowledgements}

This work was supported by the ANRT through CIFRE sponsorship 1262/2013. The authors gratefully thank Benjamin Rotenberg and Maximilien Levesque for fruitful discussions.

\section{References}

(1) Khirevich, S.; Höltzel, A.; Seidel-Morgenstern, A.; Tallarek, U. Time and Length Scales of Eddy Dispersion in Chromatographic Beds. Anal. Chem. 2009, 81, 70577066 .

(2) Khirevich, S.; Daneyko, A.; Höltzel, A.; SeidelMorgenstern, A.; Tallarek, U. Statistical analysis of packed beds, the origin of short-range disorder, and its impact on eddy dispersion. Journal of Chromatography A 2010, 1217, 4713-4722.

(3) Hlushkou, D.; Bruns, S.; Tallarek, U. Highperformance computing of flow and transport in physically reconstructed silica monoliths. Journal of Chromatography A 2010, 1217, 3674-3682.

(4) Boţan, A.; Ulm, F.-J.; Pellenq, R. J.-M.; Coasne, B. Bottom-up model of adsorption and transport in multiscale porous media. Phys. Rev. E 2015, 91, 032133.

(5) Levesque, M.; Duvail, M.; Pagonabarraga, I.; Frenkel, D.; Rotenberg, B. Accounting for adsorption and desorption in lattice Boltzmann simulations. Phys. Rev. E 2013, 88, 013308.

(6) Vanson, J.-M.; Coudert, F.-X.; Rotenberg, B.; Levesque, M.; Tardivat, C.; Klotz, M.; Boutin, A. Unexpected coupling between flow and adsorption in porous media. Soft Matter 2015, 11, 6125-6133. 
(7) Succi, S. The Lattice-Boltzmann equation for fluid dynamics and beyond; Oxford Science publications, 2001.

(8) Ladd, A. J. C.; Verberg, R. lattice-Boltzmann simulations of particle-fluid suspensions. Phys. Rev. E 2001, 104, 1191-1251.

(9) Lowe, C.; Frenkel, D. The super long-time decay of velocity fluctuations in a two-dimensional fluid. Physica A 1995, 220, 251 - 260.

(10) Lowe, C. P.; Frenkel, D. Do Hydrodynamic Dispersion Coefficients Exist? Phys. Rev. Lett. 1996, 77, 45524555.

(11) Merks, R.; Hoekstra, A.; Sloot, P. The Moment Propagation Method for Advection-Diffusion in the Lattice Boltzmann Method: Validation and P'eclet Number Limits. J. Comput. Phys. 2002, 183, $563-576$.

(12) Agarwal, S.; Verma, N.; Mewes, D. A lattice boltzmann model for adsorption breakthrough. Heat Mass Transfer 2005, 41, 843-854.

(13) Manjhi, N.; Verma, N.; Salem, K.; Mewes, D. Lattice Boltzmann modelling of unsteady-state $2 \mathrm{D}$ concentration profiles in adsorption bed. Chem. Eng. Sci. 2006, $61,2510-2521$.

(14) Zalzale, M.; McDonald, P. Lattice Boltzmann simulations of the permeability and capillary adsorption of cement model microstructures. Cem. Concr. Res. 2012, 42, $1601-1610$.

(15) Anderl, D.; Bauer, M.; Rauh, C.; Rude, U.; Delgado, A. Numerical simulation of adsorption and bubble interaction in protein foams using a lattice Boltzmann method. Food Funct. 2014, 5, 755-763.

(16) Pham, N. H.; Swatske, D. P.; Harwell, J. H.; Shiau, B.J.; Papavassiliou, D. V. Transport of nanoparticles and kinetics in packed beds: A numerical approach with lattice Boltzmann simulations and particle tracking. Heat Mass Transfer 2014, 72, 319 - 328.

(17) Hlushkou, D.; Gritti, F.; Daneyko, A.; Guiochon, G.; Tallarek, U. How Microscopic Characteristics of the Adsorption Kinetics Impact Macroscale Transport in Chromatographic Beds. J. Phys. Chem. 2013, 117, 22974-22985.

(18) Ren, J.; Guo, P.; Guo, Z.; Wang, Z. A Lattice Boltzmann Model for Simulating Gas Flow in Kerogen Pores. Transport in Porous Media 2015, 106, 285-301.

(19) Ning, Y.; Jiang, Y.; Liu, H.; Qin, G. Numerical modeling of slippage and adsorption effects on gas transport in shale formations using the lattice Boltzmann method. Journal of Natural Gas Science and Engineering 2015, 26, 345 - 355 .

(20) Long, G.; Xiao, L.; Shan, X.; Zhang, X. Modeling adsorption with lattice Boltzmann equation. Scientific Reports 2016, 6, 319 - 328 .

(21) Yang, J.; Crawshaw, J.; Boek, E. S. Quantitative determination of molecular propagator distributions for solute transport in homogeneous and heterogeneous porous media using lattice Boltzmann simulations. Water Resour. Res. 2013, 49, 8531-8538.

(22) Gray, F.; Cen, J.; Shah, S.; Crawshaw, J.; Boek, E. Simulating dispersion in porous media and the influence of segmentation on stagnancy in carbonates. $A d$ vances in Water Resources 2016, 97, 1-10.

(23) Gray, F.; Cen, J.; Boek, E. S. Simulation of dissolution in porous media in three dimensions with lattice
Boltzmann, finite-volume, and surface-rescaling methods. Phys. Rev. E 2016, 94.

(24) Vanson, J.-M.; Boutin, A.; Klotz, M.; Coudert, F.-X. Transport and adsorption under liquid flow: the role of pore geometry. Soft Matter 2017, in press, DOI: 10.1039/c6sm02414a.

(25) Zeiser, T.; Lammers, P.; Klemm, E.; Li, Y. W.; Bernsdorf, J.; Brenner, G. CFD-calculation of flow, dispersion and reaction in a catalyst filled tube by the lattice Boltzmann method. Chemical Engineering Science 2001, 56, 1697 - 1704, 16th International Conference on Chemical Reactor Engineering.

(26) Wernert, V.; Bouchet, R.; Denoyel, R. Influence of Molecule Size on Its Transport Properties through a Porous Medium. Analytical Chemistry 2010, 82, 26682679 .

(27) Marco, V. B. D.; Bombi, G. Mathematical functions for the representation of chromatographic peaks. Journal of Chromatography A 2001, 931, 1 - 30.

(28) Giddings, J. C. Dynamics of chromatography: Principles and theory; CRC Press, 2002.

(29) van Deemter, J.; Zuiderweg, F.; Klinkenberg, A. Longitudinal diffusion and resistance to mass transfer as causes of nonideality in chromatography. Chemical Engineering Science 1956, 5, 271 - 289. 
Graphical TOC Entry

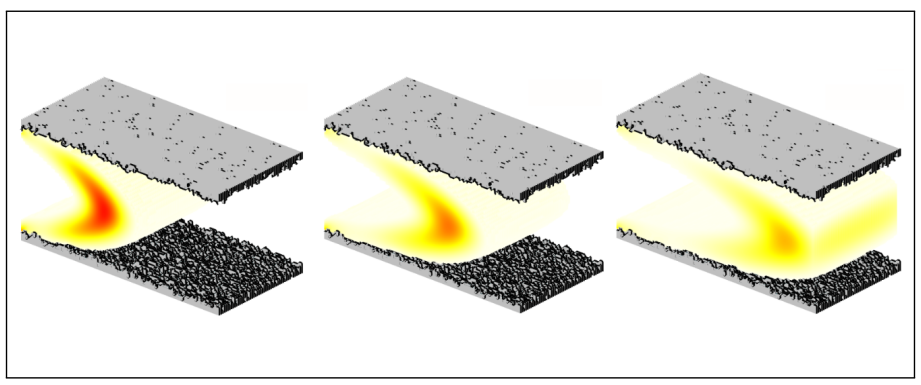

